\title{
Protective effect of polydatin on learning and memory impairments in neonatal rats with hypoxic-ischemic brain injury by up-regulating brain-derived neurotrophic factor
}

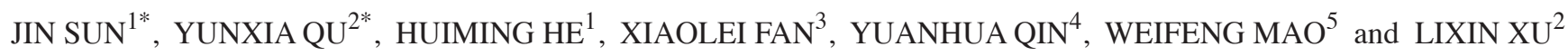 \\ ${ }^{1}$ Hospital of Maternal and Child Health of Dalian, Dalian, Liaoning 116033; ${ }^{2}$ Department of Pediatrics, \\ The Second Affiliated Hospital of Dalian Medical University, Dalian, Liaoning 116023; Departments of ${ }^{3}$ Microbiology, \\ ${ }^{4}$ Parasitology and ${ }^{5}$ Biotechnology, Dalian Medical University, Dalian, Liaoning 116044, P.R. China
}

Received November 3, 2013; Accepted May 9, 2014

DOI: $10.3892 / \mathrm{mmr} .2014 .2577$

\begin{abstract}
Polydatin is a key component of Polygonum cuspidatum, a herb with medical and nutritional value. The present study investigated the protective effect of polydatin against learning and memory impairment in neonatal rats with hypoxic-ischemic brain injury (HIBI). The unilateral common carotid artery ligation method was used to generate neonatal HIBI rats. Y-maze testing revealed that rats with HIBI exhibited memory impairment, while rats with HIBI treated with polydatin displayed enhanced long-term learning and memory. Of note, polydatin was found to upregulate the expression of hippocampal brain-derived neurotrophic factor (BDNF) in rats with HIBI. BDNF has a role in protecting HIBI-induced brain tissue injury and alleviating memory impairment. These findings showed that polydatin had a protective effect against learning and memory impairment in neonatal rats with HIBI and that the protective effect may be mediated through the upregulation of BDNF.
\end{abstract}

\section{Introduction}

Perinatal hypoxic-ischemic brain injury (HIBI) is a major cause of mortality and neurodevelopmental impairment in newborn infants (1). The increased risk of neurodevelopmental

Correspondence to: Dr Weifeng Mao, Department of Biotechnology, Dalian Medical University, Dalian, Liaoning 116044, P.R. China

E-mail: maoweifeng@dlmedu.edu.cn

Mr. Lixin Xu, Department of Pediatrics, The Second Affiliated Hospital of Dalian Medical University, Dalian, Liaoning 116023, P.R. China

E-mail: xuhans@hotmail.com

*Contributed equally

Key words: polydatin, Polygonum cuspidatum, hypoxic-ischemic brain injury, brain-derived neurotrophic factor impairment may be due to the rate of survival of premature infants, with survivors displaying neurological sequelae $(2,3)$. Thus, investigations are required to develop effective drugs to protect against neurodevelopmental impairment in newborn infants with HIBI. Polydatin (Fig. 1) is one of the primary bioactive components of Polygonum cuspidatum and has been reported to exhibit a neuroprotective effect in the rat brain, as well as protect against learning and memory impairment in a rat model of vascular dementia, alleviate oxygen and glucose deprivation-induced myocardial cell injury and attenuate ischemia-reperfusion-induced cardiac myocyte damage (4-8). However, the protective effect and mechanism of polydatin on perinatal rats with HIBI has yet to be elucidated.

Neonatal hypoxic-ischemic model rats exhibit predictable brain injuries similar to those observed clinically in humans (9-12). In the present study, a neonatal rat model of HIBI was generated using a unilateral carotid artery ligation method on postnatal seven-day-old rats, comparable to the 34-week-old human fetus (13). The protective effect of polydatin was then investigated on neurodevelopmental impairment in the neonatal rats with HIBI. The effect of polydatin on learning and memory in neonatal rats with HIBI was assessed using a behavioral test. Furthermore, the expression of brain-derived neurotrophic factor (BDNF) was assessed in the rat hippocampus following polydatin treatment. The findings of the present study may be useful to determine the potential neuroprotective effect of polydatin in neonatal rats with HIBI and to develop a potential clinical treatment of neonatal hypoxic-ischemic encephalopathy.

\section{Materials and methods}

Polydatin and experimental animals. Polydatin dry powder (Yousi Biotechnology Inc., Shanghai, China) was purified, analyzed using high-performance liquid chromatography and the purity was determined to be $>99 \%$. A total of 156 seven-day-old, healthy Sprague-Dawley rats, weighing between 12 and $19 \mathrm{~g}$, were selected regardless of gender from the Animal Experimental Center of Dalian Medical University (Animal license no: SYXK20080002; Dalian, 
China). Rats were maintained under a 12-h light/dark cycle at $22^{\circ} \mathrm{C}$ with free access to food and water.

Neonatal rats with HIBI, grouping and treatment design. The animal model of HIBI was generated using Rice's method (9). In brief, a midline incision was made at the rat neck and the subcutaneous fat was separated. The left carotid artery was exposed and permanently ligated. The rats were maintained in nitrogen gas containing $8 \%$ oxygen in closed containers for $2 \mathrm{~h}$. Following $2 \mathrm{~h}$ hypoxia, the rats were allowed to recover. Polydatin dry powder was dissolved in physiological saline and intraperitoneally injected into the rats $(10 \mathrm{mg} / \mathrm{kg}$ body weight) once a day for 10 consecutive days. A total of 156 experimental Sprague-Dawley rats were randomly divided into the following three groups: Sham-operated (SO; $\mathrm{n}=40)$, model (rats with HIBI treated with saline; $n=58$ ) and polydatin (PD; rats with HIBI treated with polydatin; $n=58$ ). The study was approved by the ethics committee of the Hospital of Maternal and Child Health of Dalian, Dalian, China.

$Y$-maze learning test. The Y-maze learning test was used to assess the learning and memory of the rats. Ten days following hypoxic-ischemic damage, rats with HIBI were tested using the stochastic restless method. The rats underwent 20 trials every $24 \mathrm{~h}$ with the Y-maze parameters (60V; $5 \mathrm{sec}$ duration). The error reaction number (ERN), total reaction time (TRT) and correct avoidance rate (CAR) were measured to assess the learning ability of the rats.

Immunohistochemical staining. Rats were sacrificed using an intracardiac perfusion fixation method. Brains were removed and fixed using $4 \%$ paraformaldehyde in phosphate-buffered saline for $48 \mathrm{~h}$. The tissues were dehydrated and embedded in paraffin for immunohistochemistry. The embedded brain tissues were sliced to a thickness of $4 \mu \mathrm{m}$. Rabbit anti-mouse BDNF antibodies (H-117; Santa Cruz Biotechnology, Inc., Santa Cruz, CA, USA) and a streptavidin-peroxidase immunohistochemistry kit (Zhongshan Jinqiao Biotechnology Co. Ltd., Beijing, China) were used for the staining. Image-Pro Plus 6 software (Media Cybernetics, Inc., Rockville, MD, USA) was used to calculate the average optical density of the staining.

Western blot analysis. Rats were sacrificed using decapitation. The left hippocampal tissues were isolated from the fresh brain tissue and stored in liquid nitrogen for western blot analysis. Total protein was extracted from the hippocampal tissues and measured. Equal aliquots of protein were used for western blot analysis using rabbit anti-mouse BDNF and $\beta$-actin antibodies (Santa Cruz Biotechnology, Inc., Santa Cruz, CA, USA). Gray value analysis software (Quantity One; Bio-Rad Inc., Hercules, CA, USA) was used to analyze the gray value of the protein bands. Relative BDNF protein expression was calculated using the ratio of BDNF gray value to $\beta$-actin gray value.

Statistical analysis. SPSS 13.0 software (SPSS, Inc., Chicago, IL, USA) was used for the statistical analyses. All experimental data are presented as the mean \pm standard deviation for each group. Differences among the groups were
Table I. Effect of polydatin on memory impairment in HIBI rats on day one assessed using the Y-maze test.

\begin{tabular}{lccrc}
\hline Groups & Samples & TRT $(\%)$ & ERN $(\%)$ & CAR $(\%)$ \\
\hline SO & 10 & $157.92 \pm 10.31$ & $2.80 \pm 1.23$ & $43.50 \pm 5.80$ \\
Model & 10 & $201.47 \pm 21.96^{\mathrm{a}}$ & $10.10 \pm 0.74$ & $21.00 \pm 6.99^{\mathrm{a}}$ \\
PD & 10 & $179.80 \pm 11.23^{\mathrm{b}}$ & $5.60 \pm 1.26$ & $29.50 \pm 4.38^{\mathrm{b}}$ \\
\hline
\end{tabular}

Data are presented as the mean \pm standard deviation. ${ }^{\mathrm{a}} \mathrm{P}<0.01$ vs. SO group; ${ }^{b} \mathrm{P}<0.01$ vs. $\mathrm{PD}$ group. HIBI, hypoxic-ischemic brain injury; TRT, total reaction time; ERN, error reaction number; CAR, correct avoidance rate; $\mathrm{SO}$, sham-operated group; $\mathrm{PD}$, polydatin group.

Table II. Effect of polydatin on memory impairment in HIBI rats on day two assessed using the Y-maze test.

\begin{tabular}{lcccc}
\hline Groups & Samples & TRT $(\%)$ & ERN $(\%)$ & CAR (\%) \\
\hline SO & 10 & $148.94 \pm 12.60$ & $2.40 \pm 0.52$ & $45.00 \pm 7.45$ \\
Model & 10 & $198.40 \pm 26.69^{\mathrm{a}}$ & $9.80 \pm 1.40$ & $18.00 \pm 7.15^{\mathrm{a}}$ \\
PD & 10 & $173.84 \pm 9.72^{\mathrm{b}}$ & $5.00 \pm 0.94$ & $31.00 \pm 8.76^{\mathrm{b}}$
\end{tabular}

Data are presented as the mean \pm standard deviation. ${ }^{\mathrm{a} P}<0.01$ vs. SO group; ${ }^{b} \mathrm{P}<0.01$ vs. PD group. HIBI, hypoxic-ischemic brain injury; TRT, total reaction time; ERN, error reaction number; CAR, correct avoidance rate; $\mathrm{SO}$, sham-operated group; $\mathrm{PD}$, polydatin group.

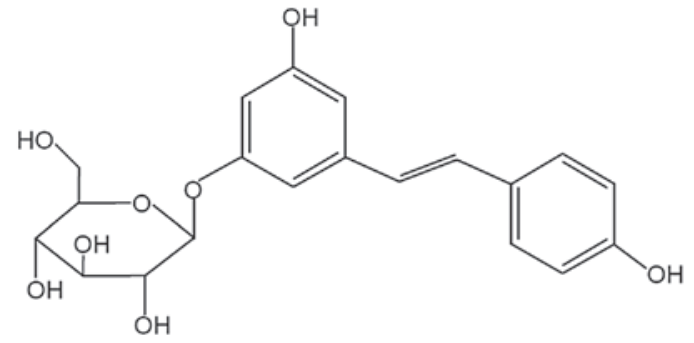

Figure 1. Molecular structure of polydatin.

compared using one-way analysis of variance (ANOVA), or multiple ANOVA followed by least significant difference tests. $\mathrm{P}<0.05$ was considered to indicate a statistically significant difference.

\section{Results}

Effect of polydatin on memory impairment in rats with HIBI. On days one and two, Y-maze testing revealed that the TRT and ERN of the rats in the model group were significantly increased $(\mathrm{P}<0.01)$, while the CAR was significantly reduced $(\mathrm{P}<0.01)$ compared with the rats in $\mathrm{SO}$ group (Tables I and II). Compared with the rats in model group, the TRT of the rats in the PD and SO groups was observed to be significantly decreased $(\mathrm{P}<0.01)$, while the CAR was found to be significantly increased $(\mathrm{P}<0.01$; Tables I and II). These findings suggested that rat memory was significantly impaired 
A

$24 \mathrm{~h}$

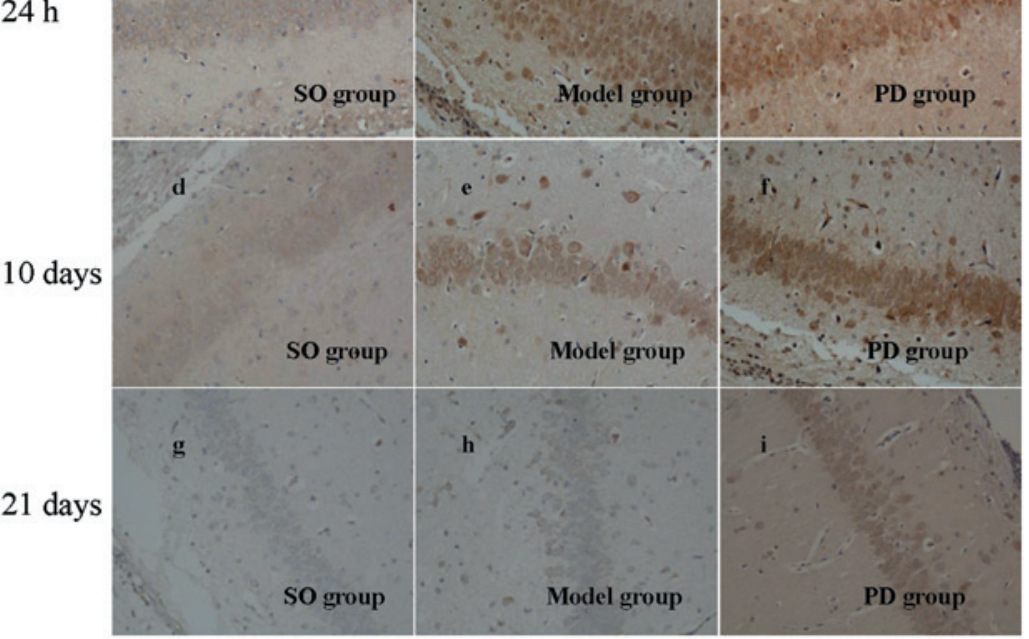

$\mathbf{B}$

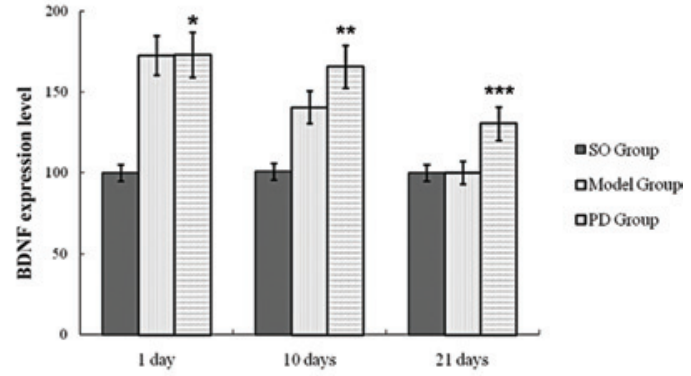

Figure 2. BDNF expression in the hippocampal CA1 region of the rats detected using immunohistochemistry. (A) Imunohistochemical staining of BDNF expression in the hippocampal CA1 region of HIBI rats in each group on day 1, 10 and 21 after hypoxic-ischemic damage (magnification, x200). (B) Immunohistochemistry scores. BDNF expression was scored between 100 and 200, with BDNF expression in the rats in the SO group set to 100. Data are presented as the mean \pm standard deviation of three independent staining experiments. $\mathrm{P}<0.01$ vs. SO group on day one; ${ }^{* *} \mathrm{P}<0.01$ vs. SO group and model group on day $10 ;{ }^{* * *} \mathrm{P}<0.01 \mathrm{vs}$. SO and model groups on day 21. HIBI, hypoxic-ischemic brain injury; BDNF, brain-derived neurotrophic factor; SO, sham-operated; PD, polydatin.

by hypoxia and ischemia and that polydatin ameliorated the HIBI-induced memory impairment.

Polydatin increases BDNF expression in the rat hippocampus. BDNF expression in the rat hippocampus was assessed using immunohistochemistry and western blot analyses. In the rats in the SO group, BDNF was observed to be expressed in the hippocampal CA1 and CA3 regions. At $24 \mathrm{~h}$ after hypoxic-ischemic damage, BDNF expression in the left hippocampal CA1 region was found to be significantly increased in the rats in the model group compared with the rats in the SO group ( $\mathrm{P}<0.01$; Fig. 2$)$. There was no significant difference in BDNF expression in the rats in the model group compared with those in the PD group $(\mathrm{P}>0.05$; Fig. 2). On days 10 and 21 following hypoxic-ischemic damage, BDNF expression in the hippocampal CA1 region in the rats in the model group was observed to be significantly increased compared with those in the $\mathrm{SO}$ group $(\mathrm{P}<0.01$; Fig. 2). Furthermore, BDNF expression in the hippocampal CA1 region of the rats in the PD group was found to be significantly increased compared with the rats in the model group ( $\mathrm{P}<0.05$; Fig. 2). BDNF expression in the hippocampal CA3 region exhibited a similar pattern of expression to that in the hippocampal CA1 area (data not shown).

Western blot analysis revealed that at $24 \mathrm{~h}$ after hypoxic-ischemic damage, BDNF expression was increased in the hippocampus of the rats in the model group compared with those in the SO group (Fig. 3). Furthermore, BDNF expression was observed to be increased in the rats in the PD group compared with those in the $\mathrm{SO}$ and model groups. On day 10 following hypoxic-ischemic damage, BDNF expression in hippocampus of the rats in the model group was found to be reduced compared with that on day one. On day 21 after hypoxic-ischemic damage, there was no significant difference in BDNF expression in the hippocampus of the
A

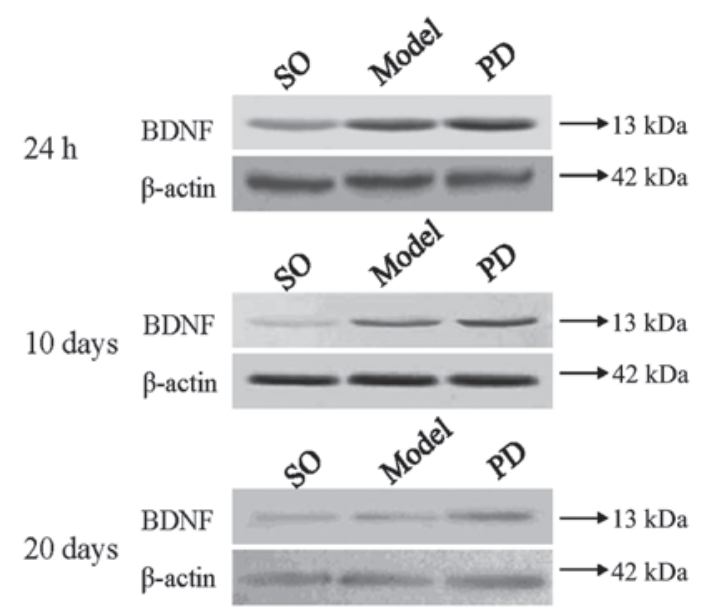

B

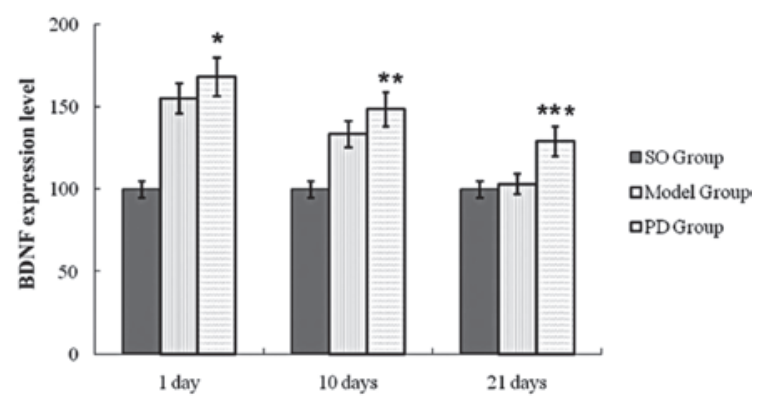

Figure 3.Western blot analysis of BDNF expression in the hippocampus of the rats in each group. (A) Western blot analysis of BDNF expression in the hippocampus of the rats in each group on day 1, 10 and 21 after hypoxic-ischemic damage. $\beta$-actin was used as an internal control. (B) Scoring of western blot analysis data using the ratio of BDNF gray value to $\beta$-actin gray value. BDNF expression was scored between 100 and 200, with BDNF expression in the rats in the SO group set to 100 . Data are presented as the mean \pm standard deviation of three independent experiments. $\mathrm{P}<0.01$ vs. SO group on day one; ${ }^{* *} \mathrm{P}<0.01$ vs. SO and model groups on day $10 ;{ }^{* * *} \mathrm{P}<0.01$ vs. SO and model groups on day 21 . BDNF, brain-derived neurotrophic factor; $\mathrm{SO}$, sham-operated group; $\mathrm{PD}$, polydatin group. 
rats in the $\mathrm{SO}$ group compared with the model group; however, BDNF expression in the rats in the PD group remained high (Fig. 3). These findings suggested that polydatin induced BDNF expression in the hippocampus of rats and sustained the expression of BDNF in rats with HIBI.

\section{Discussion}

Polydatin has been reported to have an important role in neuroprotection against cerebral cell injury induced by focal ischemic-reperfusion (8). In the present study, a neonatal HIBI rat model was used to investigate the effect of polydatin on HIBI-induced learning and memory impairment.

Y-maze tests were used to detect the spatial learning and memory of the rats $(14,15)$. Rats in the maze exhibited a passive avoidance reaction to electrical stimulation. Generally, subsequent to training, rats learn and remember the spatial location of the safe zone within the maze. However, hippocampal damage impairs memory and spatial orientation (12). In the present study, the Y-maze test results reflected HIBI-induced hippocampal neuron damage and memory impairment in the rats. Following polydatin treatment, the Y-maze test revealed that TRT and ERN were reduced, while CAR was increased, compared with the rats in the model group. These findings suggested that hypoxia and ischemia impaired learning and memory in neonatal rats and that polydatin enhanced spatial orientation and memory in rats with hypoxic-ischemic injury, as well as alleviated neurologic sequelae in rats with HIBI.

BDNF is widely distributed throughout the brain to protect against brain injury. Moreover, BDNF expression is increased in cerebral ischemia (16-18). BDNF reduces neuronal damage following ischemia, as well as promotes nerve repair (19-23). In the present study, in the rat model of HIBI, cerebral ischemia and reperfusion injury caused high BDNF expression, which is associated with local neuronal resistance to injury. Of note, BDNF exerted protective effects on spatial memory deficits following neonatal hypoxic-ischemic injury.

The present study showed that hippocampal BDNF expression was increased following HIBI, but gradually decreased to normal levels within 21 days. Furthermore, polydatin was found to have a protective effect against memory impairment in rats with HIBI and significantly increased the expression of BDNF. Thus, while the neuroprotective mechanism of polydatin in HIBI treatment requires further investigation, the high levels and duration of BDNF expression induced by polydatin may have a protective role in neurons against brain damage induced by hypoxia-ischemia. In conclusion, the present study showed that polydatin may have an important role in promoting neuronal survival and may contribute to neuron recovery following injury, as well as HIBI-induced memory impairment. Polydatin was found to increase the expression of hippocampal BDNF, which may be the long-term mechanism underlying the improvements in learning and memory induced by polydatin in rats with HIBI.

\section{Acknowledgements}

This study was supported by the Program for Liaoning Excellent Talent in University to Dr Weifeng Mao, Liaoning Science and Technology Project (nos. 2013225086 and
2011225013) and the Liaoning Medicine Engineering Project and the Dalian Science and Technology Project (no. 2010E15SF158).

\section{References}

1. Vannucci RC: Experimental biology of cerebral hypoxia-ischemia: relation to perinatal brain damage. Pediatr Res 27: 317-326. 1990.

2. Willson-Costello D, Friedman H, Minich N, Fanaroff AA, and Hack M: Improved survival rates with increased neurodevelopmental disability for extremely low birth weight infants in the 1990s. Pediatrics 115: 997-1003, 2005.

3. van Handel M, Swaab H, de Vries LS, and Jongmans MJ: Long-term cognitive and behavioral consequences of neonatal encephalopathy following perinatal asphyxia: a review. Eur J Pediatr 166: 645-654, 2007.

4. Zhao KS, Jin C, Huang X, Liu J, Yan WS, Huang Q, and Kan W: The mechanism of Polydatin in shock treatment. Clin Hemorheol Microcirc 29: 211-217, 2003.

5. Cheng Y, Zhang HT, Sun L, Guo S, Ouyang S, Zhang Y, and $\mathrm{Xu}$ J: Involvement of cell adhesion molecules in polydatin protection of brain tissues from ischemia-reperfusion injury. Brain Res 1110: 193-200, 2006.

6. Miao Q, Wang S, Miao S, Wang J, Xie Y, and Yang Q: Cardioprotective effect of polydatin against ischemia/reperfusion injury: roles of protein kinase $\mathrm{C}$ and mito K(ATP) activation. Phytomedicine 19: 8-12, 2011.

7. Ji H, Zhang X, Du Y, Liu H, Li S, and Li L: Polydatin modulates inflammation by decreasing NF-kappaB activation and oxidative stress by increasing Gli1, Ptch1, SOD1 expression and ameliorates blood-brain barrier permeability for its neuroprotective effect in pMCAO rat brain. Brain Res Bull 87: 50-59, 2012

8. Li RP, Wang ZZ, Sun MX, Hou XL, Sun Y, Deng ZF, and Xiao K: Polydatin protects learning and memory impairments in a rat model of vascular dementia. Phytomedicine 19: 677-681, 2012.

9. Rice JE, Vannucci RC, and Brierley JB: The influence of immaturity on hypoxic-ischemic brain damage in the rat. Ann Neurol 9: 131-141, 1981.

10. Vannucci RC, Connor JR, Mauger DT, Palmer C, Smith MB, Towfighi J, and Vannucci SJ: Rat model of perinatal hypoxic-ischemic brain damage. J Neurosci Res 55: 158-163, 1999.

11. Yager JY: Animal models of hypoxic-ischemic brain damage in the newborn. Semin Pediatr Neurol 11: 31-46, 2004.

12. Golan $\mathrm{H}$ and Huleihel $\mathrm{M}$ : The effect of prenatal hypoxia on brain development: short- and long-term consequences demonstrated in rodent models. Dev Sci 9: 338-349, 2006.

13. Hagberg H, Bona E, Gilland E and Puka-Sundvall M: Hypoxia-ischaemia model in the 7-day-old rat: possibilities and shortcomings. Acta Paediatr Suppl 422: 85-88, 1997.

14. Paul CM, Magda G and Abel S: Spatial memory: Theoretical basis and comparative review on experimental methods in rodents. Behav Brain Res 203: 151-164, 2009.

15. Peng S, Zhang Y, Zhang J, Wang $\mathrm{H}$ and Ren B: Effect of ketamine on ERK expression in hippocampal neural cell and the ability of learning behavior in minor rats. Mol Biol Rep 37: 3137-3142, 2010.

16. Yamada K, Mizuno M and Nabeshima T: Role for brain-derived neurotrophic factor in learning and memory. Life Sci 70 : 735-744, 2002.

17. Zheng F, Zhou X, Moon C and Wang H: Regulation of brain-derived neurotrophic factor expression in neurons. Int $\mathrm{J}$ Physiol Pathophysiol Pharmacol 4: 188-200, 2012.

18. Wang Y, Cao M, Liu A, Di W, Zhao F, Tian Y and Jia J: Changes of inflammatory cytokines and neurotrophins emphasized their roles in hypoxic-ischemic brain damage. Int J Neurosci 123: 191-195, 2013.

19. Galvin KA, and Oorschot DE: Continuous low-dose treatment with brain-derived neurotrophic factor or neurotrophin-3 protects striatal medium spiny neurons from mild neonatal hypoxia/ischemia: a stereological study. Neuroscience 118: 1023-1032, 2003. 
20. Kurozumi K, Nakamura K, Tamiya T, Kawano Y, Kobune M, Hirai S, Uchida H, Sasaki K, Ito Y, Kato K, Honmou O, Houkin K, Date I and Hamada H: BDNF gene-modified mesenchymal stem cells promote functional recovery and reduce infarct size in the rat middle cerebral artery occlusion model. Mol Ther 9: 189-197, 2004.

21. Marini AM, Jiang X, Wu X, Tian F, Zhu D, Okagaki P and Lipsky RH: Role of brain-derived neurotrophic factor and NF-kappaB in neuronal plasticity and survival: From genes to phenotype. Restor Neurol Neurosci 22: 121-130, 2004.
22. Liu L, Zhang X, Wang L, Yang R, Cui L, Li M, Du W and Wang S: The neuroprotective effects of Tanshinone IIA are associated with induced nuclear translocation of TORC1 and upregulated expression of TORC1, pCREB and BDNF in the acute stage of ischemic stroke. Brain Res Bull 82: 228-233, 2010.

23. Im SH, Yu JH, Park ES, Lee JE, Kim HO, Park KI, Kim GW, Park CI and Cho SR: Induction of striatal neurogenesis enhances functional recovery in an adult animal model of neonatal hypoxic-ischemic brain injury. Neuroscience 169: $259-268,2010$ 